\title{
L'idéal coopératif dans une organisation contemporaine
}

Le cas de la coopérative de La Louve

Cooperative ideals in a contemporary organisation

The case of the Louve cooperative

\section{El ideal cooperativo en una organización contemporánea}

El caso de la cooperativa La Louve

\section{Hajar El Karmouni et Muriel Prévot-Carpentier}

Numéro 340, avril 2016

URI : https://id.erudit.org/iderudit/1037404ar

DOI : https://doi.org/10.7202/1037404ar

Aller au sommaire du numéro

Éditeur(s)

Association Recma

ISSN

1626-1682 (imprimé)

2261-2599 (numérique)

Découvrir la revue

Citer cet article

El Karmouni, H. \& Prévot-Carpentier, M. (2016). L'idéal coopératif dans une organisation contemporaine : le cas de la coopérative de La Louve. Revue internationale de l'économie sociale, (340), 78-92.

https://doi.org/10.7202/1037404ar
Résumé de l'article

Le modèle des coopératives de consommation connaît en France un regain d'intérêt de la part de sociétaires qui souhaitent organiser des alternatives aux circuits traditionnels de consommation. Insérés, pour une grande part d'entre eux, dans le salariat classique, ces sociétaires bénévoles reproduisent leur utilisation quotidienne des outils de gestion pour construire leur projet coopératif. Nous interrogeons dans cet article les mécanismes de reproduction de l'organisation entrepreneuriale classique dans une coopérative de consommation, à travers l'étude du cas de la coopérative La Louve. Ce cas donne à voir la primauté de la dimension pratique dans sa mise en tension avec les principes coopératifs. 


\title{
L'IDÉAL COOPÉRATIF DANS UNE ORGANISATION CONTEMPORAINE LE CAS DE LA COOPÉRATIVE DE LA LOUVE
}

\author{
par Hajar El Karmouni* \\ et Muriel Prévot-Carpentier**
}

\begin{abstract}
* Institut de recherche en gestion, université Paris-Est Marne-La-Vallée.

** Laboratoire Sophiapol, université Paris-Ouest NanterreLa Défense.
\end{abstract}

\begin{abstract}
Le modèle des coopératives de consommation connaît en France un regain d'intérêt de la part de sociétaires qui souhaitent organiser des alternatives aux circuits traditionnels de consommation. Insérés, pour une grande part d'entre eux, dans le salariat classique, ces sociétaires bénévoles reproduisent leur utilisation quotidienne des outils de gestion pour construire leur projet coopératif. Nous interrogeons dans cet article les mécanismes de reproduction de l'organisation entrepreneuriale classique dans une coopérative de consommation, à travers l'étude du cas de la coopérative La Louve. Ce cas donne à voir la primauté de la dimension pratique dans sa mise en tension avec les principes coopératifs.
\end{abstract}

\section{Cooperative ideals in a contemporary organisation : The case of the Louve cooperative}

There has been renewed interest in the consumer cooperative model among members wanting to develop alternatives to conventional supply chains. Mainly conventional employees, these voluntary members replicate their everyday use of management tools to construct their cooperative project. In this article, we investigate the mechanisms for replicating the conventional entrepreneurial structure in a consumer cooperative based on the case of the Louve cooperative. This case shows how the practical dimension has more importance in the tension with cooperative principles.

\section{El ideal cooperativo en una organización contemporánea: el caso de la cooperativa La Louve} El modelo de las cooperativas de consumidores conoce un interés renovado en Francia por parte de societarios que desean organizar alternativas a los circuitos tradicionales de consumo. En la mayor parte integrados en el trabajo asalariado clásico, estos asalariados benévolos repiten el uso cotidiano de los instrumentos de gestión para construir su proyecto cooperativo. En el artículo se cuestionan los mecanismos de reproducción de la organización empresarial clásica en una cooperativa de consumidores, a través del estudio de caso de la cooperativa La Louve. Este caso deja ver la primacía de la dimensión práctica en su puesta en tensión con los principios cooperativos. 
Les évolutions récentes de certaines coopératives ont provoqué une approche nouvelle en termes de "capitalisme coopératif ", approche par ailleurs critiquée au regard des principes, de l'histoire et de la diversité du mouvement coopératif (Draperi, 2012). Cet article traite des mécanismes de reproduction de l'organisation entrepreneuriale classique au sein d'une coopérative de consommation: La Louve. Cette reproduction apparaît en distorsion avec l'idée d'une organisation du mouvement coopératif comme alternative aux modèles traditionnels de gestion et conduit à l'émergence d'un nouveau type de fonctionnement coopératif.

La présence de deux grands profils dans la structure, dont l'un se cantonne volontairement aux tâches fonctionnelles et de gestion, tandis que l'autre assume et porte en outre la charge symbolique du projet, ancre des formes de subordination volontaire dans l'activité de travail des bénévoles. Cette organisation faisant passer au second plan le principe de double qualité des coopérateurs, à la fois sociétaires et usagers, sera abordée dans une première partie, qui détaillera la manière dont se façonnent les prises de décision avec pour référentiel le travail dans sa dimension économique.

Notre deuxième partie prolongera cette question du travail bénévole dans la coopérative prenant pour modèle le travail professionnalisé, en démontrant qu'il conduit à un isomorphisme. Ce concept d'isomorphisme sera interrogé sous l'angle microsocial par le prisme de l'activité réelle que développent les membres bénévoles de La Louve avec la coopérative américaine de Park Slope Food Coop pour modèle.

Nous terminerons en pointant la primauté de la dimension pratique au détriment de la dimension politique et ses points de tension avec les principes du mouvement coopératif d'émancipation par l'activité.

\section{Encadré}

\section{La coopérative de La Louve}

La Louve est un supermarché coopératif et participatif, à but non lucratif, dont l'ouverture est prévue à la fin 2016 et qui vendra des produits de qualité à moindre coût. Les coopérateurs souhaitent reproduire dans le XVIII arrondissement de Paris le modèle original de fonctionnement de Park Slope Food, une coopérative prospère de New York, qui compte 16000 sociétaires. D’abord groupement d'achats depuis 2011, la coopérative de consommation de La Louve se double, depuis novembre 2014, d'une société par actions simplifiées (SAS) à forme coopérative, une forme juridique mieux adaptée à son gigantisme.

Comme pour son modèle américain, le rôle des membres de La Louve est central. Ils sont à la fois sociétaires*, travailleurs (à raison de trois heures par mois minimum) et acheteurs exclusifs. Le supermarché est en cours d'élaboration par ses 1456 membressouscripteurs**, qui assurent ainsi la quasi-totalité du travail.

* La souscription minimale est de dix parts à 10 euros la part ou, pour les bénéficiaires des minima sociaux, une part. Cette dernière catégorie représente 3,4 \% des 1456 souscripteurs au 24 juin 2015.

** Au 24 juin 2015. 


\section{La structuration d'une organisation coopérative d'envergure}

\section{L'idée de double qualité des membres coopérateurs mise au second plan}

La création de l'association de La Louve, en 2011, est issue de l'initiative de deux Américains vivant à Paris et souhaitant réaliser leur rêve de création d'une alternative citoyenne de taille dans la ville de la gastronomie française, avec des appuis prestigieux parmi les «meilleurs ouvriers de France » et des potagers de renom tel le potager du roi à Versailles ${ }^{(1)}$. Ces deux fondateurs sont parvenus à constituer un premier groupe de personnes autour de leur projet.

L'une des premières personnes à les avoir rejoints, devenue ensuite un pilote du projet, décrit ainsi les instances dirigeantes de la structure associative en avril 2014: «Les sept personnes qui composent le comité de pilotage de La Louve [sont] trois américains plutôt dans la vision et quatre personnes plutôt dans la gestion. Et

(1) Vidéo de présentation du projet pour la campagne de crowdfunding, surlesiteKisskissbankbank. Kisskissbankbank. com/le-supermarche-collabo ratif-de-la-louve--3 (consulté le 23 juillet 2015). les deux composantes ont besoin l'une de l'autre. Nous, sans eux, on n'aurait pas osé, mais eux, sans nous, ils ne pourraient pas faire, donc on est soudés par cette forme de complémentarité. » Cet extrait d'entretien montre que la structure est coordonnée par des pilotes qui jonglent entre «utopie », pour ceux qui sont considérés comme les « visionnaires ${ }^{(2)}$ » du projet, et «pragmatisme» par les «gestionnaires ». Deux groupes sociaux distincts sont donc aux prises au sein de cette organisation et ces deux types de profil apparaissent comme structurants pour et dans l'activité d'organisation de La Louve. Cela se matérialise par le fait que les membres de La Louve attendent des retombées sociales de leur action au sein de l'organisation, mais ne se pensent pas comme

(2) Le terme « visionnaire » a été utilisé lors des réunions d'information. Ce qualificatif apparaît également de nombreuses fois dans le corpus d'entretiens. porteurs d'une double qualité en tant que sociétaires et usagers, des usagers à la fois travailleurs et consommateurs.

\section{Méthodologie}

Cet article se base sur l'analyse des données provenant d'observations participantes et non participantes (réunions des groupes de travail, assemblées générales, réunions des pilotes, préparation et distribution de paniers, événements auxquels La Louve participe), des documents internes (site Web, comptes rendus de réunions, « Basecamp » [l'outil de travail collaboratif de La Louve]) et externes (presse, radio), ainsi que des entretiens avec les pilotes, les coordinateurs et les membres actifs. Notre analyse interdisciplinaire mêle deux disciplines, philosophie et sciences de gestion, pour comprendre un même terrain de recherche: La Louve. Cette posture théorique peut être définie comme ergologique (Schwartz, 2000), c'est-à-dire qu'elle prend pour point d'ancrage la compréhension de l'activité telle qu'elle s'organise et se réalise, ainsi que la manière dont ceux qui la portent l'explicitent et lui donnent sens. 
Les membres, qu’ils appartiennent à la catégorie des « gestionnaires » ou des " visionnaires », manifestent une croyance profonde en la force imaginative portée dans le projet par les membres fondateurs. La culture américaine est valorisée comme un élément participant de la capacité d'entreprendre et de se tourner vers l'avenir sans se laisser arrêter par des freins ou par des références historiques considérées comme passéistes.

L'observation participante nous a permis de voir émerger une sorte de discours porteur d'un mythe sur la création de la coopérative (Salmon, 2008). Pour consacrer la jeune existence de La Louve, les 148 pages du rapport d'activité 2014 ont été présentées en assemblée générale de la manière suivante: "Ça nous permet d'historiser La Louve(3)."

(3) Assemblée générale de l'association Les Amis de La Louve du 22 avril 2015, salle paroissiale Saint-Denys, Paris. Cette assertion témoigne de l'ancrage de cette association créée il y a trois ans et dont le décollage réel se situe fin 2013 suite à la campagne de crowdfunding et à l'importante couverture médiatique dont a alors bénéficié le projet.

Ce discours du mythe n'est pas propre à cette organisation et a déjà été pointé par Meyer et Rowan (1977) comme étant de l'ordre de l'activité symbolique et participant à la vie de l'organisation et à son institutionnalisation. Il est souligné comme indispensable pour différencier l'organisation concernée des organisations qui pourraient être considérées comme similaires ou du même champ (DiMaggio, Powell, 1983, p. 157), en l'occurrence les supermarchés de l'économie traditionnelle ou de type coopératif, dans le cas de La Louve.

\section{Le travail dans sa dimension économique pour référentiel}

L'organisation telle qu'elle est agencée entre les membres n'est pas sans rappeler le référentiel implicite de la grande majorité des personnes œuvrant au sein de La Louve qui reproduisent dans leur temps de travail bénévole un modèle éprouvé au sein de leur sphère professionnelle (Simonet, 2010). Le travail est abordé stricto sensu (Schwartz, 2000), exempt de l'idée de double qualité, qui imposerait de le penser du point de vue de la coopération et des valeurs véhiculées dans l'activité. Ainsi, dans cette phase d'élaboration du futur supermarché, trois éléments émergent et semblent caractéristiques du travail considéré dans sa dimension économique, auquel se réfèrent les membres bénévoles: une division du travail, un cloisonnement des tâches et le respect d'une hiérarchie informelle.

La division du travail est formalisée dans cette organisation, qui s'est dotée très tôt d'un organigramme. Dans cette phase d'intense développement, le cloisonnement des tâches est justifié par la nécessité de disposer d'un ordre de marche efficace dans une organisation de grande envergure où la quasitotalité du travail est effectuée par les bénévoles, supervisés par des pilotes et des coordinateurs également bénévoles ${ }^{(4)}$. L’organigramme présente

(4) Seule une personne était salariée au second semestre 2014. Un second salarié à plein temps a été engagé le $1^{\text {er }}$ mars 2015.

(5) Au 24 juin 2015. seize groupes de travail $^{(5)}$ et se complexifie au rythme de l'agrandissement de la structure. Chaque coordinateur prend en charge un axe particulier du travail: fonctionnement du futur magasin, approvisionnement, groupement d'achat, communication, coordination du projet (organisation-méthodes, comité ordre du jour), systèmes d'information, financement, recrutement-événements, 
participation des membres, aménagement, gestion-administration, comptabilité-gestion, juridique, écosystèmes, mobilités, vie du local.

Il faut souligner que La Louve bénéficie pour son développement des compétences organisationnelles du travail usuel accompli par les membres bénévoles dans le salariat, qui constitue pour une grande part d'entre eux leur activité principale. Les compétences professionnelles sont prioritairement valorisées. Du point de vue de l'émancipation dans l'activité prônée comme un principe cardinal du mouvement coopératif, la reproduction dans la sphère privée de l'activité accomplie dans le travail salarié n'est pas encouragée même si elle est majoritaire chez les membres.

Au sein des groupes, la prescription des tâches n'est pas dévolue au seul coordinateur: la structure La Louve affirme l'absence de pouvoir hiérarchique. Cependant, l'organisation prenant le pas et chacun voulant se rendre le plus efficace possible, la plupart des membres préfèrent qu'une tâche leur soit attribuée clairement sur le modèle du travail traditionnel. De manière marginale, certains membres évoquent même une gêne concernant la gestion de la prescription des tâches, mais ils restent très largement minoritaires. Z. l'exprime ainsi: "J'ai l'impression que tu fais cela plutôt en entreprise, tu vois de... Tu as une tâche à faire, tu as ton poste et, derrière... ton $n+1$ va valider que tu as bien fait ce que... ce qu'on t'a demandé. A La Louve... c'est plutôt... Enfin, je ne le vois pas comme cela. Je le vois comme [...] on donne ce qu'on a, mais on n'est pas en train de... se juger et de se donner des... des... Enfin si, on se donne des tâches à faire, mais [...] je crois que c'est la manière dont c'est fait qui est un peu moyenne, quoi.»

Ainsi, une conception hiérarchique des rapports sociaux, sur le modèle de l'entreprise où la subordination est contractuelle (Friot, 2012), est soulignée dans ce verbatim et observée dans plusieurs situations d'activité dans la coopérative. Cela apparaît assez discordant avec la conception du rapport égalitaire à autrui portée par le mouvement coopératif. Cela ne signifie toutefois pas qu'une évolution des rapports ne puisse pas intervenir progressivement par l'éducation à la coopération que voudraient entamer des membres de la coopérative et par la démultiplication des dimensions du projet.

\section{Le travail comme signe de l'engagement}

De manière générale, les membres actifs au sein de La Louve valorisent la capacité à s'engager sans compter dans l'activité de travail. M. dit par exemple: "Moi, j'ai... Toute ma vie, j'ai été une workaholic, tu ne peux pas imaginer. Moi... Moi, toute ma vie, c'était du boulot. »Cette dimension de l'engagement, du mérite à travailler est centrale dans l'imaginaire collectif que construisent les membres de La Louve. Le travail en général et le travail bénévole accompli dans le projet commun sont extrêmement valorisés.

Une importance toute particulière doit être conférée à cet imaginaire qui se forge en commun lorsque l'on rappelle que l'organisation sociale et économique de La Louve est basée sur le travail des membres. Le président de la coopérative, parlant d'une membre très active, coordinatrice d'un groupe, la décrit en disant: "Elle travaille plus de 35 heures pour son boulot, mais elle travaille encore plus de 35 heures pour La Louve, c'est incroyable. »Cette dimension d'engagement apparaît comme un moyen de trouver du sens 
par le biais d'une activité bénévole promouvant une consommation responsable (Özçaglar-Toulouse, 2009).

Du point de vue du travail, un autre aspect doit alors être considéré. Le dépassement des trois heures de travail mensuelles prévues pour chaque membre dans le projet entérine l'engagement que chacun d'entre eux consent à investir pour faire aboutir le projet commun en vue de sa réussite. Le fait d'être prêt à offrir de son temps pour se mettre au service de La Louve est l'un des comportements régulièrement observés. Parlant d'un membre qui se présente pour être élu au conseil d'administration, le président de l'association dit: "Il y aura un changement dans sa vie professionnelle et donc il va avoir plus de temps, qu'il souhaite consacrer à La Louve(6). »

(6) Assemblée générale de la coopérative La Louve du 22 avril2015, salle paroissiale Saint-Denys, Paris.
Cet argument est mis en avant comme un élément favorable. La disponibilité pour le projet rend l'engagement patent.

Un dernier aspect concerne l'engagement sans mesure, en plus de l'imaginaire du travail sans limites et de la capacité d'engagement dans l'activité. Cet engagement apparaît comme un moyen d'accession au pouvoir, que ce soit pour les bénévoles ou pour les salariés, et confère aux membres qui y consentent une capacité décisionnelle dans le projet. Les membres concernés par ce fort engagement ne le développent pas pour acquérir cette capacité décisionnelle et établir volontairement une hiérarchie. Ils ne souhaitent nullement en formaliser une et continuent de se prévaloir d'un fonctionnement autogestionnaire.

De plus, la capacité décisionnelle est également influée par le capital social des membres, qui leur permet de savoir valoriser leurs compétences professionnelles et personnelles dans l'organisation ou de faire connaître un réseau social pouvant être utile pour le développement du projet de La Louve.

Le travail vivant, réel, duquel naissent toutes sortes d'adaptations nouvelles, était un enjeu social très présent dans le mouvement coopératif français. Ainsi, Gaumont (1921) décrit concrètement la manière dont les coopérateurs se rencontrent et élaborent leurs projets coopératifs parisiens, vivent des difficultés. Ce volet du travail coopératif dans son contenu, sa dimension sociale, portant l'empreinte des valeurs véhiculées par ses membres, apparaît relativement impensé dans ce nouveau type de coopérative, plus axé sur une conception du travail en volume, du moins dans cette phase de développement. A ce stade du projet se dégage une prégnance de la reproduction du référentiel du travail professionnalisé ordinaire, de la dimension économique du travail, et des formes de domination traditionnelles dans les organisations, dues au présentéisme ou au groupe social auquel les personnes appartiennent.

\section{Une organisation empreinte d'isomorphisme}

Pour analyser l'organisation telle qu'elle fonctionne dans la phase de création du supermarché collaboratif de La Louve, nous proposons un " retravail » de la notion d'"isomorphisme institutionnel » forgée par DiMaggio et Powell (1983) et déjà appliquée au secteur associatif (Enjolras, 1996; Bidet, 2003). Cette notion d'isomorphisme institutionnel fait ressortir trois dimensions, que Bidet expose ainsi quant à leur influence pour le cas 
des associations: "Un isomorphisme normatif qui résulte de la professionnalisation des associations, un isomorphisme coercitif résultant de l'influence des pouvoirs publics, un isomorphisme mimétique que traduit le recours à des solutions éprouvées pour répondre aux problèmes donnés » (Bidet, 2003, p. 166). La spécificité du retravail que nous proposons est de donner à voir les effets dans l'activité de cette notion macrosociale. Il s'agit bien d'importer au niveau microsocial un concept jusqu'alors principalement en usage au niveau macrosocial.

\section{La reproduction d’un modèle coopératif éprouvé aux Etats-Unis}

La coopération mise en place par La Louve est inspirée de son modèle américain, la Park Slope Food Coop. Son succès est systématiquement rappelé à chaque réunion d'information proposée aux nouveaux adhérents, ainsi que dans les vidéos de promotion du futur supermarché et les échanges avec les médias.

Le manuel des membres rédigé par la Park Slope Food Coop est intégralement repris au sein de La Louve pour organiser le travail, dans un premier temps. Ainsi, le groupe «Fonctionnement du futur magasin » commence d'ores et déjà à créer les tutoriels prévus pour que chacun puisse «occuper son rôle dans le futur supermarché » ${ }^{(7)}$. Cette formalisation de la prescription prend pour modèle le travail des squad leaders dans la coopérative américaine, c'est-à-dire que la coopération est pensée sur le mode de l'utilité, sur le modèle américain de la participation de bénévoles

(7) Réunion des coordinateurs du 23 mars 2015, local de la Goutte-d'Or, La Louve, Paris. du tiers secteur, et non en fonction du modèle français marqué, comme l'écrit Espagne, par la "conviction que seule la coopérative de consommation est porteuse de possibilités d'amélioration du sort des travailleurs, que seule elle incarne, avec la primautédu consommateur, un authentique projet coopératif» (Espagne, 2006 [1996], p. 19). Ceci est à relier à la culture américaine sous-jacente au projet, insufflée par les deux fondateurs et confortée par les liens avec la Park Slope Food Coop.

La structuration de l'activité est en effet préétablie et consolidée sans cesse avec leurs homologues d'outre-Atlantique. Les membres fondateurs de La Louve échangent au moins une fois par semaine avec les membres fondateurs et dirigeants de la coopérative new-yorkaise sur les procédures mises en place, les erreurs à éviter, les chiffres du futur supermarché, par exemple sur les comparaisons de surface, mais aussi sur les débats en cours. Le coordinateur du groupe juridique a réaffirmé l'importance tutélaire du modèle à l'occasion d'une assemblée générale ${ }^{(8)}$ : "On ne va pas réinventer l'eau chaude, on a le modèle de Park Slope. » Il faut souligner l'absence de questionnement sur l'intégration des spécificités culturelles sous-jacentes au contexte français.

(8) Assemblée générale du 21 octobre 2014, salle SaintBruno, Paris.

\section{Une volonté de mimétisme}

Lors de leur travail bénévole, les membres de La Louve font un usage intense des modes de communication en vigueur dans les entreprises auxquelles ils appartiennent en tant que salariés: outils de gestion de projet, brainstorming, diagramme de Gantt, PowerPoint, etc. Ces comportements, 
calqués sur le modèle entrepreneurial classique, peuvent s'apparenter à de l'isomorphisme "mimétique ou managérial» (DiMaggio, Powell, 1983). Or, dans l'activité, cet isomorphisme révèle une ambivalence que nous pouvons donner à voir à travers l'exemple du fonctionnement du comité "Ordre du jour ». Celui-ci est à la fois un garant de la démocratie et un frein au deuxième principe coopératif de l'Alliance coopérative internationale sur le pouvoir démocratique des membres. Il est directement inspiré de la Park Slope Food Coop et permet d'avoir une instance de traitement de toute question de membre avant les assemblées générales. Cette reproduction du modèle paraît garantir la possibilité de voir surgir toute question sans en exclure aucune. Dans les faits, cependant, elle exige une forte mobilisation du membre pour faire émerger un débat et interroge sur les entraves générées par la modélisation forte de la participation. La procédure prévue par le comité est en effet assez lourde. Un membre souscripteur lambda souhaitant voir traitée une question devra compter environ six mois pour qu'elle puisse être mise au vote.

Pour donner à voir comment se déroule le processus, exposons la question posée succinctement par une membre lors de l'assemblée générale du 24 février 2015 concernant «la proposition d'extension du droit à n'acheter qu'une part », c'est-à-dire l'extension à deux nouvelles catégories de bénéficiaires du droit à souscrire à la coopérative de La Louve proposée aux minima sociaux. Cette question a été exposée, une fois travaillée, par cette membre lors de l'assemblée générale du 22 avril 2015, durant près d'une demi-heure. Sa présentation, très pédagogique et documentée, abordait l'impact de cet élargissement sur les fonds propres de la coopérative, la volonté d'éviter toute discrimination ou exclusion au sein de La Louve, etc. Elle a été votée partiellement lors de l'assemblée générale du 25 juin 2015, car un volet de la question restait insuffisamment documenté.

Cet exemple de l'émergence et du traitement d'une question de fonctionnement relativement anodine nous semble marquer la prégnance de l'isomorphisme mimétique et de ses limites dans l'organisation de La Louve.

Par ailleurs, la pratique du benchmarking, courante au sein de La Louve, renforce la reproduction des formes traditionnelles du marché dans cette phase de développement. Cette expansion, dirigée sur le modèle d'autres organisations du même champ, va dans le sens de ce que Huault (2009) affirme: "Le benchmarking peut être appréhendé comme l'institutionnalisation d'un processus mimétique, puisqu'il consiste à se comparer aux concurrents et à s'inspirer de leurs recettes. " La coopérative parisienne a bénéficié notamment du partage de l'expérience du réseau Biocoop et met régulièrement en avant des comparaisons avec Les Nouveaux Robinson, notamment en ce qui concerne le nombre de références au mètre carré.

Cependant le modèle principal de comparaison reste la Park Slope Food Coop, ce qui peut être considéré comme une forme d'intercoopération dans l'activité et non plus comme le benchmarking que promeuvent les grandes entreprises à forme capitalistique. En effet, dans ces entreprises, cette pratique abstrait totalement le milieu de travail comme totalité dont les processus s’interpénètrent. Seul un élément de l'organisation 
est extrait, le processus de stockage par exemple, sans considérer l'activité ou le projet social du secteur concerné (Bruno, Didier, 2013). Or, le benchmarking tel qu’il se pratique dans cette expérience coopérative se traduit bien plus comme de l’intercoopération. Souvent considérée à un niveau plus global dans le mouvement coopératif, l'intercoopération est pratiquée dans bien des constructions de projet coopératif de manière informelle lorsqu'elle est regardée à hauteur d’activité réelle des membres. Cette intercoopération active qui traverse l’Atlantique entre La Louve et la Park Slope Food Coop est une duplication se voulant complète du modèle et non une partition de l'activité au profit d'une organisation ad hoc d'un secteur donné et circonscrit. La frontière entre modèle coopératif et modèle capitaliste traditionnel apparaît dans cette pratique qui, regardée trop rapidement, pourrait être abusivement caractérisée comme étant du benchmarking.

\section{Débats et pratiques: une recherche de sûreté}

Un débat extrêmement clivé a eu lieu lors de l'été 2014. La question fondamentale était la répartition des tâches dans l'association, notamment de déterminer qui allait occuper le poste de coordinateur polyvalent et réaliser la tâche centrale d'élaboration du business plan. Le débat a été tranché dans un premier temps par un refus du repli sur le modèle entrepreneurial, au motif que la professionnalisation des activités, propre à l'isomorphisme normatif, augurait mal de la suite du projet de supermarché collaboratif, basé sur l'investissement bénévole de ses membres.

Ainsi, un isomorphisme coercitif avait été identifié par les membres fondateurs venus des Etats-Unis comme dangereux et explicitement dénoncé comme pervers par le président de l'association, en juillet 2014, car il ferait par la suite "coller» les innovations de la Louve au "catalogue de subventions » proposées par la puissance publique. Même s'ils ne le revendiquent pas comme tel, cette volonté d'indépendance correspond à un principe central du mouvement coopératif. Le quatrième principe de l'Alliance coopérative internationale, intitulé "Autonomie et indépendance ", précise: "La recherche de fonds à partir de sources extérieures doit se faire dans des conditions qui préservent le pouvoir démocratique des membres et maintiennent l'indépendance de leur coopérative.»

Cependant, la possibilité de financement public partiel de la rémunération d'emploi, à travers le dispositif de subvention, a finalement été mise en place avec un "emploi-tremplin » qui a débuté le $1^{\text {er }}$ mars 2015. Comme identifié dans d'autres projets, «les subventions publiques [...] n'exercent pas de pressions coercitives sur l'agent. Elles comportent cependant des incitations qui peuvent se traduire par des effets d'éviction au détriment des ressources volontaires »(Enjolras, 2008, p. 29). Ainsi, les tâches du poste salarié ont été définies à partir des contraintes imposées, pour pouvoir en bénéficier par le conseil régional, pourvoyeur du dispositif: par exemple, devoir dresser une fiche de poste explicitant l'ensemble des tâches du salarié employé sur ce contrat.

L'isomorphisme normatif comme recherche de sûreté apparaît toujours présent entre les deux groupes sociaux qui structurent l'activité de La Louve: les « visionnaires » et les "gestionnaires ». Les " gestionnaires » 
sont la plupart du temps dans du normatif, alors que les "visionnaires » y ont ponctuellement recours. Ces derniers semblent parfois faire appel à des normes de gestion, par exemple pour reporter l'éventualité de la mise en place d'un service de livraison extrêmement défendu par le porteur de l'idée. Lorsque les idées ne sont pas tout à fait conformes à leur conception actuelle du projet ou pourraient mettre en péril son développement rapide

(9) Réunion des coordinateurs du 23 mars 2015, local de la Goutte-d'Or, La Louve, Paris. et réaliste, ils les caractérisent, par exemple pour la livraison, comme "trop complexe» et "trop individualisante, surtout à ce stade d'élaboration du projet ${ }^{(9)}$.

\section{Entreprendre autrement?}

\section{Dimension politique}

La Louve s’inscrit dans le mouvement coopératif, du point de vue du sociétariat et de la participation active de ses membres, ainsi que sur le volet de son inscription comme alternative aux modèles de distribution conventionnels. Ce dernier volet rejoint partiellement le deuxième des trois critères de Prades, qui estime que "la meilleure façon de résister au formatage des consciences, à la dictature de l'avoir et à la puissance de la normalisation est de donner des illustrations concrètes des prémices de la "coopérativisation" du monde" (Prades, 2015, p. 45). Le « formatage des consciences » et la « puissance de la normalisation » sont intrinsèquement repoussés par le mouvement coopératif, qui veut stimuler une émancipation dans l'activité, à la fois individuelle et sur le plan de pratiques.

Nous pouvons souligner que le modèle coopératif français paraissait promouvoir cette émancipation par l'idée de démocratie, dans un paradigme deweyen de la démocratie comme forme de vie: "Il ne saurait y avoir d'éducation véritable que si chacun peut être reconnu comme un acteur responsable du processus de définition des buts à atteindre et des politiques à mettre en ouvre, au sein des groupes sociaux auquel il appartient. Ce n'est qu'à cette condition que l'on peut parler de démocratie » (Dewey, 2014 [1920]). Or, le renouvellement du modèle démocratique, avec une forme de coopération mettant à distance la double qualité de ses membres, conduit à un glissement vers une "démocratie libérale " telle que l'identifiait Frère (2013, p. 220), avec la délégation du pouvoir par la représentativité d'un élu, en l'occurrence le président, à La Louve. Elle se double d'une certaine atomisation des individus, c'est-à-dire de fonctionnements collaboratifs ponctuels dans lesquels chacun préserve son degré d'engagement et ses choix de tâches à effectuer dans la phase actuelle du projet.

Ce fonctionnement est accentué par une forte interchangeabilité des personnes qui peut être volontaire ou non. M. l'exprime de la manière suivante: "Je n'ai pas d'autre pouvoir que de faire de la coordination, c'est-à-dire de transmettre de l'information. [...] C'est-à-dire qu'en fait, je suis interchangeable. [...] Je ne suis pas investie d'un pouvoir. Je n'ai pas le pouvoir de dire: "Toi, tu fais cela. Toi, tu fais cela." Non! Si je pense que cela reflète l'esprit du groupe... C'est une espèce de leader démocratique d'une certaine façon, tu vois.» 
Cette volonté d'interchangeabilité, trouvant sa source dans le manque de temps ou peut-être aussi dans la volonté d'économie de soi, se traduit également dans l'activité par une absence de dessein d'exercice du pouvoir.

En effet, le contexte d'accélération de la temporalité, défini par Rosa (2010) comme étant un rapport au temps sous le signe du manque, apparaît peu favorable au développement d'un dialogue démocratique preneur de temps. Il n'est pas non plus propice au déploiement de travaux sur le long terme ou à l'emprunt de parcours sinueux, d'où pourraient émerger des gisements créatifs.

Cette nouvelle forme de coopération apparaît très libérale au sens où elle se base sur une activité individuelle considérée comme « libre » et ne pense pas le travail collectif, au détriment du déploiement dans son fonctionnement de la double qualité des sociétaires-usagers. A titre d'exemple, la liberté prise dans la réalisation des tâches au sein du groupe " Aménagement » n'a pas permis d'accomplir le travail de maîtrise d'ouvrage; un cabinet d'architectes a donc finalement été missionné pour le faire, en lieu et place des bénévoles initialement prévus. Cela a un coût très élevé pour la coopérative, environ $10 \%$ du total des fonds nécessaires au projet. Ainsi, l'absence de centration sur la dimension sociale du projet, corrélative au délaissement du sens politique de la coopération, a des répercussions sur la dimension économique du projet coopératif.

\section{Dimension institutionnelle}

Aujourd'hui les "mots de l' "entreprendre autrement" " et au premier rang celui d'"entreprise sociale", concept qui met en tension deux termes qui ne paraissaient initialement pas faits pour aller ensemble, s'il témoignent d'un "flou conceptuel [...] souligné par de nombreux chercheurs ", ne s'opposent pas à l'émergence d'une "culture renouvelée portée par de très nombreux militants engagés dans l'amélioration concrète de notre société et qui espèrent contribuer, plus loin, à la construction d'un autre monde possible» (Rousseau, 2009, p. 282). Il semble qu'au nom de cette culture renouvelée et de l'amélioration concrète, à travers laquelle la construction d'un autre monde possible et l'horizon émancipateur, la notion d'imaginaire aux potentialités multiples (Poirier, 2003) soit remise à plus tard.

C'est une question connexe que posait déjà Blanchet avec le concept d'« entre-prise» (Blanchet, 2013), soulignant, dans un paradigme assez sociologique, les interactions entre les acteurs du marché. Selon le chercheur, ces interactions constituent des contraintes pour la dimension solidaire ou équitable du projet; nous ajouterions: pour sa dimension démocratique. Les membres de La Louve qui souhaitent faire de la coopérative un "vrai » supermarché, et entrer dans un secteur déjà bien investi par l'économie traditionnelle, semblent s'être progressivement astreints à se conformer à des modes d'organisation éprouvés.

Ainsi, la difficulté à faire entrer un imaginaire différent semble inhérente au projet, puisque le référentiel est le modèle de distribution traditionnel ou des réseaux en partie coopératifs s'en approchant. Les coopérateurs veulent constituer une alternative tout en se conformant à des abaques usuels, 
notamment quant à la surface du futur magasin, au nombre de références en stock, au type de produits indispensables, au niveau de prix à obtenir pour que le « décrochage » promis par la coopérative soit réel, etc.

\section{Dimension pratique: vers un nouveau profil d'individu coopérateur?}

Bager avait déjà conceptualisé, dans le cas des coopératives scandinaves, un déclin des membres ayant un profil politique ou idéologique, au profit d'une attitude de "consommateurs" (1994, p. 47). Cette prégnance de la dimension pratique au détriment de la dimension coopératrice plus politique est une caractéristique dont les membres de La Louve voudraient pouvoir se prémunir du point de vue de la coopération: "On veut zéro client à l'ouverture de La Louve [...], on veut des coopérateurs ", dit le président ${ }^{(10)}$.

(10) Assemblée générale du 21 octobre 2014, salle SaintBruno, Paris.
Dans les faits, la majorité des membres de La Louve refusent une assimilation à un profil de militant, et le fait d'avoir «un discours idéologique » est exprimé comme un repoussoir. Cette expression est régulièrement utilisée lors des présentations en réunion d'information des nouveaux membres et pendant les assemblées générales.

Pour exemple, M., qui se dit workaholic et vivant sa première expérience coopérative à plus de 50 ans, déclare ne pas considérer La Louve comme une association, mais comme un "projet social». A ses yeux, un projet réussi implique nécessairement une organisation de type bureaucratique pour pouvoir fonctionner. Cette membre justifie son absence d'engagement antérieur par la nécessité d'une organisation solide pour garantir l'efficacité: "A chaque fois que j'ai...je...je...je me suis approchée d'associations, eh bien j'ai vu ses dysfonctionnements et je me suis... je me suis sauvée en courant. » $M$. affirme explicitement son refus de la complexité et du modèle ancien de la coopération: «Mes... mes parents étaient militants... socialistes. Moij’ai... j’ai baignélà-dedans depuis que je suis née. Mon père, il est militant, militant, tu ne peux pas savoir. Et puis en plus ilsétaient... ils étaient cathos de gauche, donc moi j'ai...j'ai vu...j'ai vu tout cela. Et, à chaque fois, il y avait toujours... c'était très... c'était toujours très... la régulation des groupes était toujours très compliquée. Donc je... je... je... volontairement, je ne me suis pas... je ne me suis pas approchée.»

Cette expression apparaît caractéristique de la primauté de la dimension pratique, mais aussi de la volonté de neutraliser le conflit dans les motivations des nouveaux profils de membres coopérateurs, comme cela a déjà été conceptualisé dans d'autres secteurs de l'économie sociale et solidaire: "C'est l'activité et non plus le projet qui fonde l'objet sur lequel se contractualisent les attentes de chacun " (Rousseau, 2009, p. 276). C'est de manière assumée que les fondateurs et membres de La Louve se réclament de leur souhait de faire usage du futur supermarché, à distance d'un désir de grand projet émancipateur. Leur objectif, à la fois principal et fédérateur, est de faire aboutir l'acte d'achat de produits de qualité à moindre coût, sans obligation d'élargir la perspective à un projet social de plus grande ampleur ou qui pourrait être porteur d'effets positifs, essaimés dans le corps social de manière imprévisible. La primauté de la dimension pratique est considérée comme favorable pour le développement de la mixité sociale; c'est ce qu'exprime Y., 
une des membres, lorsqu'elle parle de ses origines et de son amitié avec des personnes défavorisées: "Nous on est d'une famille de commerçants, si je suis venue, c'est pour que Mimi puisse faire ses courses, qu'on puisse tous manger de bons produits, de qualité [...] parce que tu comprends, Mimi il mange vraiment n’importe quoi, jamais de légumes. »

Par conséquent, ce nouveau modèle coopératif paraît faire primer l'efficacité et non mettre les principes coopératifs de prises de décisions partagées et d'égalité au premier plan, sans les récuser pour autant, dans une volonté de voir aboutir le projet. En témoigne la manière dont le président de la coopérative communique explicitement dans les médias, même dans ceux ayant une approche alternative, en disant: «In fine, plus on est pragmatique, plus le projet devient idéaliste» (Renard, 2014). Cette forme de coopération contemporaine apparaît donc renouveler assez profondément le discours qui a marqué le mouvement coopératif français et l'histoire sociale du mouvement des coopératives de consommation.

\section{Conclusion}

Selon Frère (2013), « la grammaire de l'engagement alternatif [...] n'est pas radicalement neuve par rapport à celle des engagements passés : il s'agit toujours de se réclamer des mêmes invariants: convivialité vs déshumanisation, créativité artistique vs standardisation de l'activité, autogestion vs hiérarchie, démocratie locale directe vs délégation ». Ces catégories générales éclairent bien le débat global sur le modèle coopératif français, mais ne permettent pas de saisir ce qui se passe réellement dans l'activité de travail. A travers les observations de notre terrain de recherche, l'analyse permet de donner à voir comment le monde contemporain transforme le rapport des individus à la coopération.

Le panorama du fonctionnement organisationnel de La Louve fait ressortir une prégnance de l'isomorphisme conduisant à une forme de recul des principes sociaux régissant le mouvement coopératif. Ce recul apparaît déterminé par la mise à distance du principe de double qualité délégué à ceux qui sont identifiés comme les "visionnaires " dans le projet coopératif. Porteurs des valeurs incorporées et défendues par la suite, aussi bien par les «gestionnaires» que par l'ensemble des membres, ils favorisent la dimension économique de la coopération au détriment de celles, sociale et politique, qui sont intrinsèques au mouvement des coopératives de consommation françaises. L’espace discursif est remis à plus tard, dans cette phase d'élaboration, car les « visionnaires » assument la charge du caractère symbolique du projet. Les principes coopératifs paraissent alors compatibles avec l'empreinte permanente de l'imaginaire du travail, dans sa dimension économique, et de son efficacité productive. Le travail est ainsi organisé, avec une parcellisation des tâches et une hiérarchie informelle dans l'activité qui rendent difficiles, pour le moment, la survenance de diversité d'idées, points de vue divergents, souffles en vents contraires. L'isomorphisme est assumé, ne serait-ce que par la conformité, présentée comme nécessaire du point de vue économique, à un modèle opérant de coopérative, afin de pouvoir développer le projet de supermarché collaboratif dans tout son gigantisme. 
Dans cette contribution, l'utilisation du concept institutionnaliste d'isomorphisme, au-delà de l'importation d'un concept macrosocial à une échelle microsociale, permet d'incarner les principes du mouvement coopératif dans la complexité des pressions sociales, économiques, culturelles vécues au quotidien par les individus et les collectifs dans lesquels ils s'inscrivent. C'est ce que nous donnent à voir les membres de La Louve dans la création de leur projet, apparaissant ainsi concrétiser « le scénario noir » contre lequel alertait Desroche à propos des coopératives de consommation, "mouvement [...] en train de perdre ce qui fait un mouvement: sa mémoire, sa conscience et son imagination » (Desroche, cité par Prades, 2007). 


\section{BIBLIOGRAPHIE}

Bager T., 1994, " Isomorphic processes and the transformation of cooperatives ", Annals of Public and Cooperative Economics, $\mathrm{n}^{\circ} 65$, p. 35-57.

Blanchet V., 2013, « Le commerce équitable à l'épreuve de la mode. Le rôle de la critique dans la formation des marchés ", thèse de doctorat en sciences de gestion, Paris, université Paris-Dauphine.

Bidet E., 2003, " L'insoutenable grand écart de l'économie sociale. Isomorphisme institutionnel et économie solidaire ", Revue du Mauss, $\mathrm{n}^{\circ}$ 21, p. 162-178.

Bruno I., Didier E., 2013, Benchmarking. L'Etat sous pression statistique, Paris, Zones. Dewey J., 2014 [1920], Reconstruction en philosophie, Paris, Gallimard.

DiMaggio P. J., Powell W. W., 1983, « The Iron Cage Revisited: Institutional Isomorphism and Collective Rationality in Organizational Fields ", American Sociological Review, vol. 48, $\mathrm{n}^{\circ} 2$, p. 147-160.

Draperi J.-F., 2012, La république coopérative, Bruxelles, Larcier.

Enjolras B., 1996, « Associations et isomorphisme institutionel », Recma, ${ }^{\circ}$ 261, p. 68-76. Enjolras B., 2008, « Fondements normatifs des organisations d'économie sociale et solidaire et évaluation du point de vue des politiques publiques ", Economie et Solidarités, vol. 39, n ${ }^{\circ}$, p. 14-34.

Espagne F., 2006 [1996], "Histoire, problèmes et projets de la coopération ouvrière de production en France», site de la CGScop. Les-scop.coop/export/sites/default/fr/_media/ documents/histoire-probleme-projet-cooperation-ouvriere.pdf (consulté le 2 janvier 2016). Frère B., 2013, « Faire de l'économie alternative et solidaire. Une vieille histoire », in Frère B., Jacquemain M. (coord.), Résister au quotidien, Paris, Les Presses de Science Po, p. 189-221.

Friot B., 2012, L'enjeu du salaire, Paris, La Dispute.

Gaumont J., 1921, Les sociétés de consommation à Paris: un demi-siècle d'action sociale par la coopération, Paris, Fédération des coopératives de la région parisienne.

Huault I., 2009, «Paul DiMaggio et Walter Powell. Des organisations en quête de légitimité ", in Charreire-Petit S., Huault I. (coord.), Les grands auteurs en management, Paris, EMS.

Özçaglar-Toulouse N., 2009, « Quel sens les consommateurs donnent-ils à leur consommation? Une approche par les récits de vie ", Recherches et Applications en marketing, 24 (3), p. 3-22.

Meyer, J., Rowan B., 1977, «Institutionalized Organizations: Formal Structure as Myth and Ceremony ». American Journal of Sociology, ${ }^{\circ} 83$, p. 340-364.

PoirierN.,2003, «CorneliusCastoriadis.L’imaginaire radical », RevueduMauss, n²1, p. 383-404. Prades J., 2007, « Les coopératives de consommation françaises à l'épreuve du temps ", Toulouse, blog Cerises. Blogs.univ-tlse2.fr/ cerises/files/contributions-2007-2008/2007e-Prades-sur-Desroche.pdf (consulté le 20 octobre 2015).

Prades J., 2015, Comment résister au capitalisme? Tous en coopératives!, Toulouse, Le Vent se lève.

Renard J.-C., 2014, « Un petit projet utile », Politis, n 1331, p. 14-15.

Rosa H., 2010, Accélération. Une critique sociale du temps, coll. "Théorie critique", Paris, La Découverte.

Rousseau F., 2009, « De l'association dissociée à l'entrepreneuriat social ", in Laville J.-L., Glemain P., L'économie sociale et solidaire aux prises avec la gestion, Paris, Desclée de Brouwer, p. 245-289.

Salmon C., 2008, Storytelling. La machine à fabriquer des histoires et à formater les esprits, Paris, La Découverte.

Schwartz Y., 2000, Le paradigme ergologique ou un métier de philosophe, Toulouse, Octarès.

Simonet M., 2010, Le travail bénévole. Engagement citoyen ou travail gratuit?, Paris, La Dispute. 